\title{
Sufficiency Criteria for a Class of $p$-Valent Analytic Functions of Complex Order
}

\author{
Muhammad Arif \\ Department of Mathematics, Abdul Wali Khan University, Mardan 23200, Pakistan \\ Correspondence should be addressed to Muhammad Arif; marifmaths@yahoo.com
}

Received 21 December 2012; Accepted 26 February 2013

Academic Editor: Fuding Xie

Copyright (c) 2013 Muhammad Arif. This is an open access article distributed under the Creative Commons Attribution License, which permits unrestricted use, distribution, and reproduction in any medium, provided the original work is properly cited.

In the present paper, we consider a subclass of $p$-valent analytic functions and obtain certain simple sufficiency criteria by using three different methods for the functions belonging to this class. Many known results appear as special consequences of our work.

\section{Introduction}

Let $A_{p}(n)$ be the class of functions $f(z)$ analytic and $p$-valent in the open unit disk $\mathbb{U}=\{z:|z|<1\}$ and of the form

$$
f(z)=z^{p}+\sum_{k=p+n}^{\infty} a_{k} z^{k} \quad(p \in \mathbb{N}) .
$$

In particular, $A_{p}(1)=A_{p}, A_{1}(n)=A(n)$, and $A_{1}(1)=A$. By $\mathcal{S}_{p}^{*}(n, b)$ and $\mathscr{C}_{p}(n, b), n, p \in \mathbb{N}$ and $b \in \mathbb{C} \backslash\{0\}$, we mean the subclasses of $A_{p}(n)$ which are defined, respectively, by

$$
\begin{gathered}
\operatorname{Re}\left\{1+\frac{1}{p+b-1}\left(\frac{z f^{\prime}(z)}{f(z)}-p\right)\right\}>0, \quad(z \in \mathbb{U}), \\
\operatorname{Re}\left\{1+\frac{1}{p+b-1}\left(\frac{z f^{\prime \prime}(z)}{f^{\prime}(z)}-p+1\right)\right\}>0, \quad(z \in \mathbb{U}) .
\end{gathered}
$$

For $b=1, p=1, n=1$, the previous two classes defined in (2) reduce to the well-known classes of starlike and convex, respectively.

For functions $f(z), g(z) \in A_{p}(n)$ of the form (1), we define the convolution (Hadamard product) of $f(z)$ and $g(z)$ by

$$
(f \star g)(z)=z^{p}+\sum_{k=p+n}^{\infty} a_{k} b_{k} z^{k}, \quad(z \in \mathbb{U}) .
$$

Now we define the subclass $\mathscr{M}_{p}(n, b ; g(z))$ of $A_{p}(n)$ by

$$
\operatorname{Re}\left\{1+\frac{1}{p+b-1}\left(\frac{z(f(z) \star g(z))^{\prime}}{f(z) \star g(z)}-p\right)\right\}>0
$$

$$
(z \in \mathbb{U}) \text {. }
$$

Sufficient conditions were studied by various authors for different subclasses of analytic and multivalent functions, for some of the related work see [1-8]. The object of the present paper is to obtain sufficient conditions for the subclass $\mathscr{M}_{p}(n, b ; g(z))$ of $A_{p}(n)$. We also consider some special cases of our results which lead to various interesting corollaries and relevances of some of these with other known results also being mentioned.

We will assume throughout our discussion, unless otherwise stated, that $n \in \mathbb{N}, p \in \mathbb{N}, b \in \mathbb{C} \backslash\{0\}$.

\section{Preliminary Results}

To obtain our main results, we need the following Lemma's.

Lemma 1 (see [9]). If $q(z) \in A(n)$ with $n \geq 1$ and satisfies the condition

$$
\left|q^{\prime}(z)-1\right|<\frac{n+1}{\sqrt{(n+1)^{2}+1}}(z \in \mathbb{U}),
$$


then

$$
q(z) \in \mathcal{S}^{*}(n, 1) .
$$

Lemma 2 (see [10]). If $q(z) \in A(n)$ satisfing the condition

$$
\left|\arg q^{\prime}(z)\right|<\frac{\pi}{2} \delta_{n} \quad(z \in \mathbb{U})
$$

where $\delta_{n}$ is the unique root of the equation

$$
2 \tan ^{-1}\left[n\left(1-\delta_{n}\right)\right]+\pi\left(1-2 \delta_{n}\right)=0,
$$

then

$$
q(z) \in \mathcal{S}^{*}(n, 1) .
$$

Lemma 3 (see [11]). Let $\Omega$ be a set in the complex plane $\mathbb{C}$, and suppose that $\Psi$ is a mapping from $\mathbb{C}^{2} \times \mathbb{U}$ to $\mathbb{C}$ which satisfies $\Psi(i x, y, z) \notin \Omega$ for $z \in \mathbb{U}$ and for all real $x, y$ such that $y \leq$ $(-n / 2)\left(1+x^{2}\right)$. If $q(z)=1+c_{n} z^{n}+\cdots$ is analytic in $\mathbb{U}$ and $\Psi\left(q(z), z q^{\prime}(z), z\right) \in \Omega$ for all $z \in \mathbb{U}$, then $\operatorname{Re} q(z)>0$.

\section{Main Results}

Theorem 4. If $f(z) \in A_{p}(n)$ satisfies

$$
\begin{aligned}
& \mid\left(\frac{f(z) * g(z)}{z^{p}}\right)^{1 /(p+b-1)}\left\{\frac{z(f(z) * g(z))^{\prime}}{f(z) * g(z)}+b-1\right\} \\
& \quad-(p+b-1) \mid \\
& <\frac{n+1}{\sqrt{(n+1)^{2}+1}}|p+b-1| \quad(z \in \mathbb{U}),
\end{aligned}
$$

then $f(z) \in \mathscr{M}_{p}(n, b ; g(z))$.

Proof. Let us set a function $p(z)$ by

$$
\begin{aligned}
p(z) & =z\left(\frac{f(z) * g(z)}{z^{p}}\right)^{1 /(p+b-1)} \\
& =z+\frac{a_{n+p} b_{n+p}}{(p+b-1)} z^{n}+\cdots
\end{aligned}
$$

for $f(z) \in A_{p}(n)$. Then clearly (11) shows that $p(z) \in A(n)$.

Differentiating (11) logarithmically, we have

$$
\frac{p^{\prime}(z)}{p(z)}=\frac{1}{p+b-1}\left[\frac{f(z) * g(z)^{\prime}}{f(z) * g(z)}-\frac{p}{z}\right]+\frac{1}{z}
$$

which gives

$$
\begin{aligned}
& \left|p^{\prime}(z)-1\right| \\
& =\mid\left(\frac{f(z) * g(z)}{z^{p}}\right)^{1 /(p+b-1)} \frac{1}{p+b-1} \\
& \quad \times\left\{\frac{z(f(z) * g(z))^{\prime}}{f(z) * g(z)}+b-1\right\}-1 \mid .
\end{aligned}
$$

Thus using (10), we have

$$
\left|p^{\prime}(z)-1\right| \leq \frac{n+1}{\sqrt{(n+1)^{2}+1}}, \quad(z \in \mathbb{U}) .
$$

Hence, using Lemma 1 , we have $p(z) \in \mathcal{S}^{*}(n, 1)$. From (12), we can write

$$
\frac{z p^{\prime}(z)}{p(z)}=\frac{1}{p+b-1}\left[\frac{z(f(z) * g(z))^{\prime}}{f(z) * g(z)}-p\right]+1 .
$$

Since $p(z) \in \mathcal{S}^{*}(n, 1)$, it implies that $\operatorname{Re}\left(z p^{\prime}(z) / p(z)\right)>0$. Therefore, we get

$$
\begin{aligned}
& \operatorname{Re}\left\{1+\frac{1}{p+b-1}\left(\frac{z(f(z) * g(z))^{\prime}}{f(z) * g(z)}-p\right)\right\} \\
& \quad=\operatorname{Re} \frac{z p^{\prime}(z)}{p(z)}>0,
\end{aligned}
$$

and this implies that $f(z) \in \mathscr{M}_{p}(n, b ; g(z))$.

Setting $n=p=1$ and $g(z)=z /(1-z)$ in Theorem 4, we get the following.

Corollary 5. If $f(z) \in A$ satisfies

$$
\left|\left(\frac{f(z)}{z}\right)^{1 / b}\left\{\frac{z f^{\prime}(z)}{f(z)}+b-1\right\}-b\right|<\frac{2|b|}{\sqrt{5}} \quad(z \in \mathbb{U}),
$$

then $f(z) \in \mathcal{S}^{*}(b)$, the class of starlike functions of complex order $b$.

Putting $n=p=1$ and $g(z)=z /(1-z)^{2}$ in Theorem 4, we have the following.

Corollary 6. If $f(z) \in A$ satisfies

$$
\left|\left(f^{\prime}(z)\right)^{(1-b) / b}\left\{z f^{\prime \prime}(z)+b f^{\prime}(z)\right\}-b\right|<\frac{2|b|}{\sqrt{5}} \quad(z \in \mathbb{U}),
$$

then $f(z) \in \mathscr{C}(b)$, the class of convex functions of complex order $b$.

Remark 7. If we put $b=1-\alpha$ in Corollaries 5 and 6 , we get the results proved by Uyanik et al. [1]. Furthermore, for $b=1$, we obtain the results studied by Mocanu [2] and Nunokawa et al. [3], respectively. Also if we set $b=1-\alpha$ with $g(z)=z^{p} /\left(1-z^{n}\right)$ and $g(z)=z^{p} /\left(1-z^{n}\right)^{2 p}$ in Theorem 4, we obtain the results due to Goyal et al. [4].

Theorem 8. If $f(z) \in A_{p}(n)$ satisfies

$$
\begin{aligned}
& \mid \arg \left(\frac{f(z) * g(z)}{z^{p}}\right)^{1 /(p+b-1)} \\
& \quad+\arg \left\{\frac{1}{p+b-1}\left(\frac{z(f(z) * g(z))^{\prime}}{f(z) * g(z)}+b-1\right)\right\} \mid \\
& <\frac{\pi}{2} \delta_{n} \quad(z \in \mathbb{U}),
\end{aligned}
$$

where $\delta_{n}$ is the unique root of $(8)$, then $f(z) \in \mathscr{M}_{p}(n, b ; g(z))$. 
Proof. Let $p(z)$ be given by (11), which clearly belongs to the class $A(n)$.

Now differentiating (11), we have

$$
\begin{aligned}
p^{\prime}(z)= & \left(\frac{f(z) * g(z)}{z^{p}}\right)^{1 /(p+b-1)} \\
& \times \frac{1}{p+b-1}\left\{\frac{z(f(z) * g(z))^{\prime}}{f(z) * g(z)}+b-1\right\}
\end{aligned}
$$

which gives

$$
\begin{aligned}
& \left|\arg p^{\prime}(z)\right| \\
& =\mid \arg \left(\frac{f(z) * g(z)}{z^{p}}\right)^{1 /(p+b-1)} \\
& \quad+\arg \left\{\frac{1}{p+b-1}\left(\frac{z(f(z) * g(z))^{\prime}}{f(z) * g(z)}+b-1\right)\right\} \mid .
\end{aligned}
$$

Thus using (19), we have

$$
\left|\arg p^{\prime}(z)\right| \leq \frac{\pi}{2} \delta_{n} \quad(z \in \mathbb{U}),
$$

where $\delta_{n}$ is the root of (8). Hence, using Lemma 2, we have $p(z) \in \mathcal{S}^{*}(n, 1)$.

From (20), we can write

$$
\frac{z p^{\prime}(z)}{p(z)}=\frac{1}{p+b-1}\left[\frac{z(f(z) * g(z))^{\prime}}{f(z) * g(z)}-p\right]+1 .
$$

Since $p(z) \in \mathcal{S}^{*}(n, 1)$, it implies that $\operatorname{Re}\left(z p^{\prime}(z) / p(z)\right)>0$. Therefore, we get (16), and hence $f(z) \in \mathscr{M}_{p}(n, b ; g(z))$.

Making $n=1, b=1-\alpha$ with $0 \leq \alpha<p$ and $g(z)=$ $z^{p} /(1-z)$, we have the following.

Corollary 9. If $f(z) \in A_{p}$ satisfies

$$
\begin{aligned}
& \left|\arg \left(\frac{f(z)}{z^{p}}\right)+(p-\alpha) \arg \left\{\frac{z f^{\prime}(z)}{f(z)}-\alpha\right\}\right| \\
& \quad<\frac{\pi}{2} \delta_{1}(p-\alpha) \quad(z \in \mathbb{U}),
\end{aligned}
$$

where $\delta_{1}$ is the unique root of (8) with $n=1$, then $f(z) \in$ $\delta_{p}^{*}(\alpha)$, the class of $p$-valent starlike functions of order $\alpha$.

Also if we take $n=1, b=1-\alpha$ with $0 \leq \alpha<p$ and $g(z)=z^{p} /(1-z)^{2 p}$ in Theorem 8 , we obtain the following result.

Corollary 10. If $f(z) \in A_{p}$ satisfies

$$
\begin{aligned}
& \left|\arg \left(\frac{f^{\prime}(z)}{p z^{p-1}}\right)+(p-\alpha) \arg \left\{\frac{z f^{\prime \prime}(z)}{f^{\prime}(z)}+1-\alpha\right\}\right| \\
& \quad<\frac{\pi}{2} \delta_{1}(p-\alpha) \quad(z \in \mathbb{U}),
\end{aligned}
$$

where $\delta_{1}$ is the unique root of (8) with $n=1$, then $f(z) \in$ $\mathscr{C}_{p}(\alpha)$, the class of $p$-valent convex functions of order $\alpha$.
Remark 11. For putting $p=1, \alpha=0$ in Corollary 10 and $p=$ 1 in Corollary 9, we obtain the results proved by Mocanu [10] and Uyanik et al. [1], respectively.

Theorem 12. If $f(z) \in A_{p}(n)$ satisfies

$$
\begin{aligned}
& \operatorname{Re}\left[\frac{1}{p+b-1}\left\{\frac{z(f(z) * g(z))^{\prime}}{f(z) * g(z)}\left(\rho \frac{z(f(z) * g(z))^{\prime \prime}}{(f(z) * g(z))^{\prime}}+1\right)\right\}\right. \\
& +b-1]>\frac{M^{2}}{4 L}+N
\end{aligned}
$$

where $0 \leq \rho \leq 1$ and

$$
\begin{aligned}
& L=\rho\left(p+\operatorname{Re} b-1+\frac{n}{2}\right), \\
& \begin{aligned}
& M=2 \rho \operatorname{Im} b, \\
& N=\rho((\left((\operatorname{Re} b)^{2}-(\operatorname{Im} b)^{2}-\operatorname{Re} b\right) \\
& \quad \times(p+\operatorname{Re} b-1)+(\operatorname{Im} b)^{2}(2 \operatorname{Re} b-1) \\
&\left.\left.\quad \times\left((p+\operatorname{Re} b-1)^{2}+(\operatorname{Im} b)^{2}\right)^{-1}\right)-\frac{n}{2}\right),
\end{aligned}
\end{aligned}
$$

then $f(z) \in \mathscr{M}_{p}(n, b ; g(z))$.

Proof. Let us set

$$
\frac{z(f(z) * g(z))^{\prime}}{f(z) * g(z)}=(p+b-1) p(z)-b+1 .
$$

Then $p(z)$ is analytic in $\mathbb{U}$ with $p(0)=1$.

Taking logarithmic differentiation of (28) and then by simple computation, we obtain

$$
\begin{aligned}
\frac{1}{p+b-1}\{ & \frac{z(f(z) * g(z))^{\prime}}{f(z) * g(z)} \\
& \left.\times\left(\rho \frac{z(f(z) * g(z))^{\prime \prime}}{(f(z) * g(z))^{\prime}}+1\right)+b-1\right\} \\
= & A z p^{\prime}(z)+B p^{2}(z)+C p(z)+D \\
= & \Psi\left(p(z), z p^{\prime}(z), z\right)
\end{aligned}
$$

with

$$
\begin{aligned}
& A=\rho, \quad B=\rho(p+b-1), \\
& C=-2 \rho b+\rho+1, \quad D=\frac{\rho b^{2}-\rho b}{p+b-1} .
\end{aligned}
$$

Now for all real $x$ and $y$ satisfying $y \leq-(n / 2)\left(1+x^{2}\right)$, we have

$$
\Psi(i x, y, z)=A y-B x^{2}+C(i x)+D
$$


Reputing the values of $A, B, C, D$ and then taking real part, we obtain

$$
\begin{aligned}
\operatorname{Re} \Psi(i x, y, z) & \leq-L x^{2}+M x+N \\
& =-\left(\sqrt{L x}-\frac{M}{2 \sqrt{L}}\right)^{2}+\frac{M^{2}}{4 L}+N \\
& <\frac{M^{2}}{4 L}+N,
\end{aligned}
$$

where $L, M, N$ are given in (27).

Let $\Omega=\left\{w: \operatorname{Re} w>\left(M^{2} / 4 L\right)+N\right\}$. Then $\Psi\left(h(z), z h^{\prime}(z)\right.$, $z) \in \Omega$ and $\Psi(i x, y, z) \notin \Omega$, for all real $x$ and $y$ satisfying $y \leq$ $-(n / 2)\left(1+x^{2}\right), z \in \mathbb{U}$. Using Lemma 3, we have Re $p(z)>0$. This implies that

$$
\operatorname{Re}\left\{1+\frac{1}{p+b-1}\left(\frac{z(f(z) * g(z))^{\prime}}{f(z) * g(z)}-p\right)\right\}>0,
$$

and hence $f(z) \in \mathscr{M}_{p}(n, b ; g(z))$.

If we put $p=n=1, b=1-\alpha$ and $g(z)=z /(1-z)^{2(1-\alpha)}$ in Theorem 12, we obtain the following result proved in [12].

Corollary 13. If $f(z) \in$ A satisfies

$$
\begin{gathered}
\operatorname{Re}\left\{\frac{z f^{\prime}(z)}{f(z)}\left(\rho \frac{z f^{\prime \prime}(z)}{f^{\prime}(z)}+1\right)\right\} \\
>\alpha \rho\left(\alpha-\frac{1}{2}\right)+\left(\alpha-\frac{\rho}{2}\right),
\end{gathered}
$$

then $f(z) \in \mathcal{S}^{*}(\alpha)$.

Furthermore, for $\alpha=0$ in Corollary 13, we have the following result proved in [13].

Corollary 14. If $f(z) \in$ A satisfies

$$
\operatorname{Re}\left\{\frac{z f^{\prime}(z)}{f(z)}\left(\rho \frac{z f^{\prime \prime}(z)}{f^{\prime}(z)}+1\right)\right\}>-\frac{\rho}{2}
$$

then $f(z) \in \mathcal{S}^{*}$.

\section{Acknowledgment}

The author is thankful to the Prof. Dr. Ihsan Ali, Vice chancellor of Abdul Wali Khan University Mardan, for providing research facilities in AWKUM.

\section{References}

[1] N. Uyanık, M. Aydogan, and S. Owa, "Extensions of sufficient conditions for starlikeness and convexity of order $\alpha$," Applied Mathematics Letters, vol. 24, no. 8, pp. 1393-1399, 2011.

[2] P. T. Mocanu, "Some starlikeness conditions for analytic functions," Revue Roumaine de Mathématiques Pures et Appliquées, vol. 33, no. 1-2, pp. 117-124, 1988.

[3] M. Nunokawa, S. Owa, Y. Polattoglu, M. Caglar, and E. Yavuz Duman, "Some sufficient conditions for starlikeness and convexity," Turkish Journal of Mathematics, vol. 34, no. 3, pp. 333337, 2010.
[4] S. P. Goyal, S. K. Bansal, and P. Goswami, "Extensions of sufficient conditions for starlikeness and convexity of order $\alpha$ for multivalent function," Applied Mathematics Letters, vol. 25, no. 11, pp. 1993-1998, 2012.

[5] H. Al-Amiri and P. T. Mocanu, "Some simple criteria of starlikeness and convexity for meromorphic functions," Mathematica, vol. 37, no. 60, pp. 11-20, 1995.

[6] M. Arif, I. Ahmad, M. Raza, and K. Khan, "Sufficient condition of a subclass of analytic functions defined by Hadamard product," Life Science Journal, vol. 9, no. 4, pp. 2487-2489, 2012.

[7] M. Arif, M. Raza, S. Islam, J. Iqbal, and F. Faizullah, "Some sufficient conditions for spirallike functions with argument properties," Life Science Journal, vol. 9, no. 4, pp. 3770-3773, 2012.

[8] B. A. Frasin, "Some sufficient conditions for certain integral operators," Journal of Mathematical Inequalities, vol. 2, no. 4, pp. $527-535,2008$

[9] P. T. Mocanu and Gh. Oros, "A sufficient condition for starlikeness of order $\alpha$," International Journal of Mathematics and Mathematical Sciences, vol. 28, no. 9, pp. 557-560, 2001.

[10] P. T. Mocanu, "Some simple criteria for starlikeness and convexity," Libertas Mathematica, vol. 13, pp. 27-40, 1993.

[11] S. S. Miller and P. T. Mocanu, "Differential subordinations and inequalities in the complex plane," Journal of Differential Equations, vol. 67, no. 2, pp. 199-211, 1987.

[12] V. Ravichandran, C. Selvaraj, and R. Rajalaksmi, "Sufficient conditions for starlike functions of order $\alpha$," Journal of Inequalities in Pure and Applied Mathematics, vol. 3, no. 5, pp. 1-6, 2002.

[13] J.-L. Li and S. Owa, "Sufficient conditions for starlikeness," Indian Journal of Pure and Applied Mathematics, vol. 33, no. 3, pp. 313-318, 2002. 


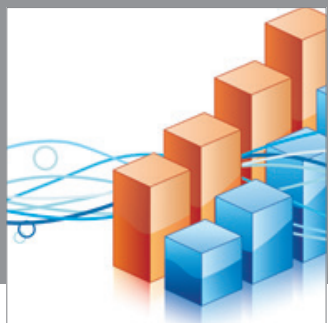

Advances in

Operations Research

mansans

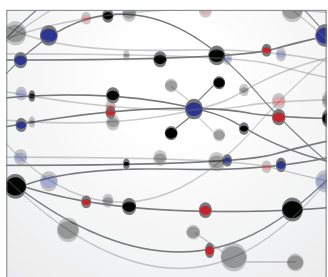

The Scientific World Journal
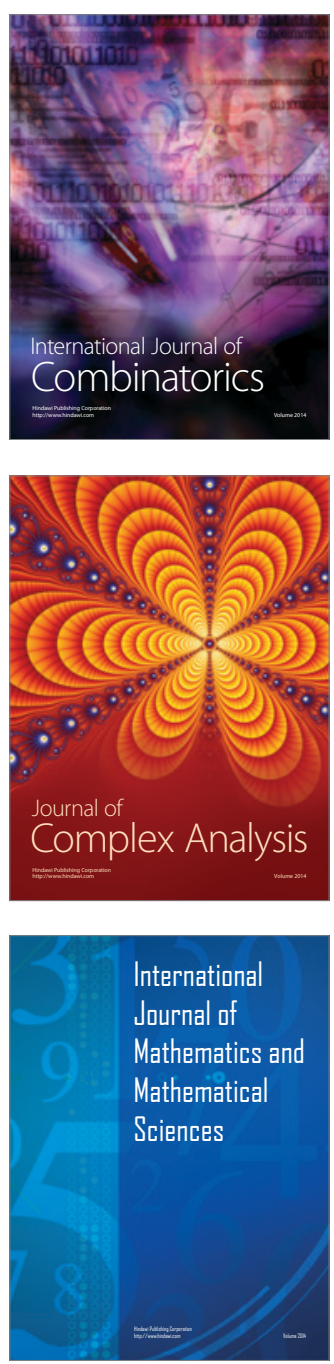
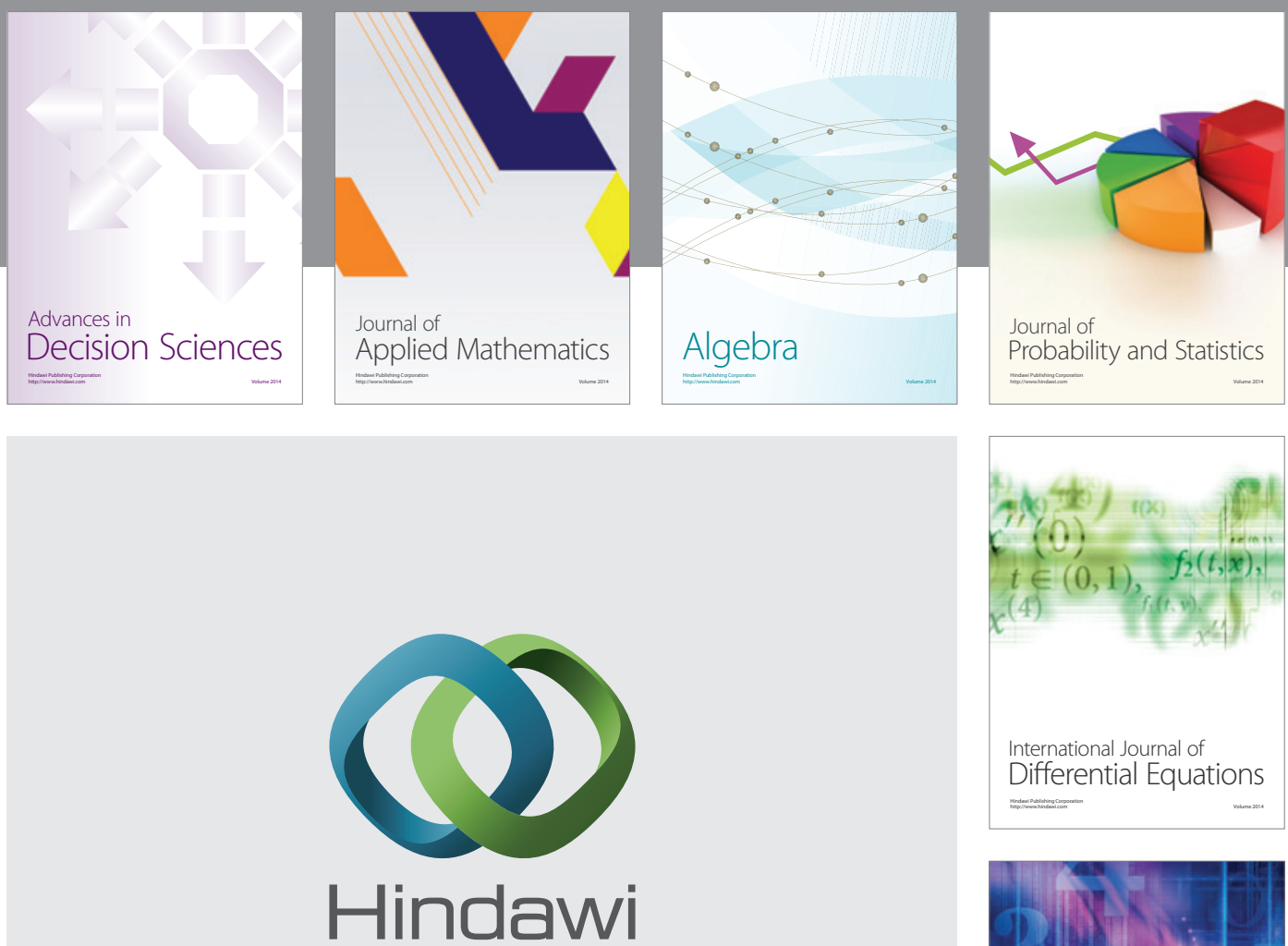

Submit your manuscripts at http://www.hindawi.com
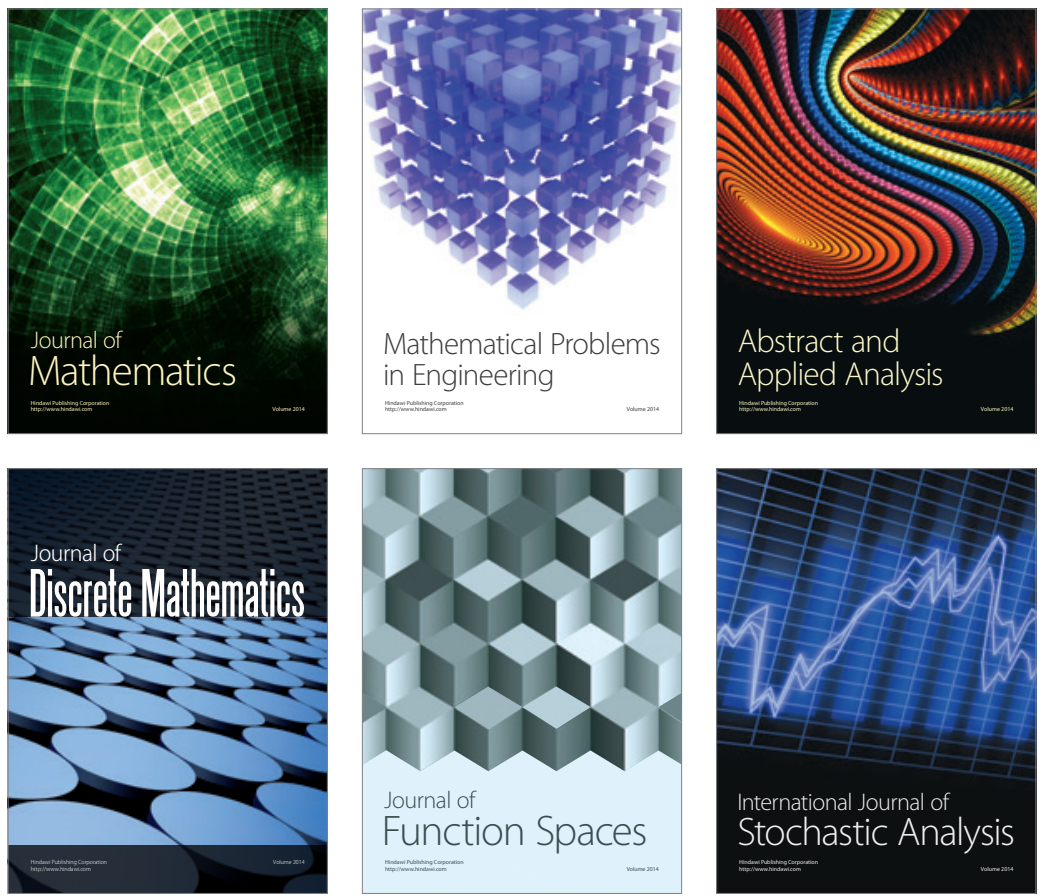

Journal of

Function Spaces

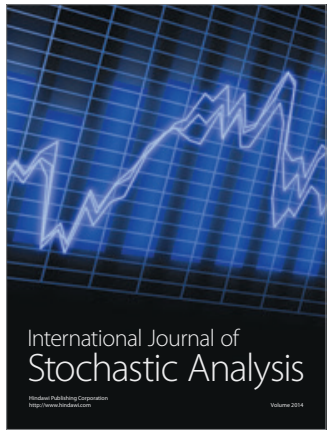

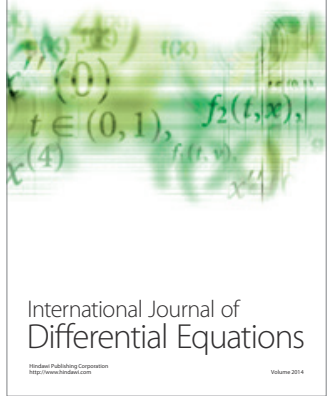
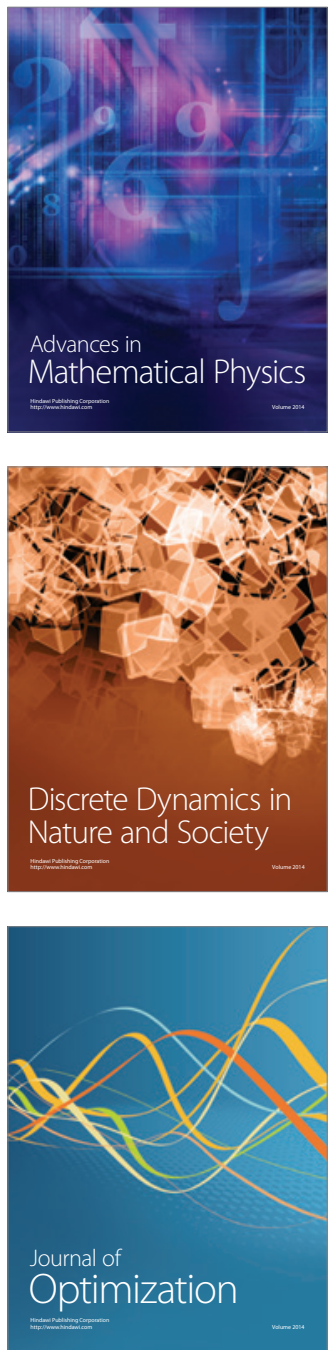